\title{
Physics Beyond the Standard Model at LHeC
}

\author{
Emmanuelle Perez \\ CERN, PH Department \\ Geneva 23 CH-1211 Switzerland
}

\begin{abstract}
Future discoveries at the LHC may call for a future DIS programme at very high energy. This could be realised by colliding one of the LHC proton beams with a new lepton beam in the LHC tunnel. Example cases are shown where high energy ep data would be needed to study and fully interpret new phenomena that the LHC may discover. The consequences, for the LHC discovery potential, of our current limited knowledge of proton structure are also shortly addressed.
\end{abstract}

\section{Introduction}

A Large Hadron Electron Collider (LHeC), allowing ep or $e A$ collisions to be performed in the LHC tunnel, is one of the possible options for the LHC upgrade. Its physics case and a possible design have been described in [1]. With a $70 \mathrm{GeV}$ electron beam in collision with one of the LHC hadron beams, a centre-of-mass energy $\sqrt{s}$ of $1.4 \mathrm{TeV}$ would be achieved, allowing deep-inelastic scattering (DIS) reactions to be studied for momentum transfer $Q^{2}$ up to $10^{6} \mathrm{GeV}^{2}$ and for Bjorken $\mathrm{x}$ values down to $10^{-6}$ for $Q^{2}>1 \mathrm{GeV}^{2}$. Low- $x$ physics at $\mathrm{LHeC}$ is described elsewhere in these proceedings [2]. In the $e p$ mode, the luminosity of this collider could reach $10^{33} \mathrm{~cm}^{-2} \mathrm{~s}^{-1}$ (i.e. an integrated luminosity of about $10 \mathrm{fb}^{-1}$ per year) such that rare, yet unobserved, phenomena may be studied in detail, as the production of electron-quark resonances. The high luminosity would also allow for a much better determination of the proton parton distribution functions at large $x$ as described elsewhere in these proceedings [3], which may be needed for a full interpretation of the discoveries that will, hopefully, be made at the LHC.

\section{Leptoquarks and supersymmetry}

An $e p$ collider, providing both baryonic and leptonic quantum numbers in the initial state, is ideally suited to a study of the properties of new bosons possessing couplings to an electron-quark pair. Such particles can be squarks in supersymmetry with $R$-parity $\left(R_{p}\right)$ violation, or first-generation leptoquark (LQ) bosons which appear naturally in various unifying theories beyond the Standard Model (SM). In $e p$ collisions, they are produced as single $s$-channel resonances via the fusion of incoming electrons with quarks in the proton. They are generically referred to as "leptoquarks" in what follows.

The single LQ production cross section depends on the unknown coupling $\lambda$ of the LQ to the electron-quark pair. For a coupling $\lambda$ of $\mathcal{O}(0.1)$, LQ masses up to about $1 \mathrm{TeV}$ could be probed at the LHeC. In $p p$ interactions, such leptoquarks would be mainly pair produced and masses up to about 1.5 to $2 \mathrm{TeV}$ can be probed at the LHC, independently of the coupling $\lambda$. However, the determination of the quantum numbers of this new particle in the pair-production mode is not possible (e.g. for the fermion number) or ambiguous and model-dependent (e.g. for the spin). Single LQ production is much better suited for such studies. As an example, the determination of the LQ fermion number $F$ via single LQ production is described below. 
Single LQ production at the LHC mainly proceeds via gluon-quark reactions [4]. The cross-section is however much lower than that at $\mathrm{LHeC}$ as shown in Fig. 1. The single production of a $F=0(|F|=2)$ LQ at the LHC, followed by the $\mathrm{LQ}$ decay into $e^{+} q$ or $e^{-} \bar{q}$ $\left(e^{-} q\right.$ or $\left.e^{+} \bar{q}\right)$, could allow the fermion number of a first generation LQ to be determined, by comparing the signal crosssections with an $e^{+}$and an $e^{-}$coming from the resonant state. This comes from the fact that the parton densities for $u$ and $d$ at high $x$ are much larger than those for $\bar{u}$ and $\bar{d}$. However, the asymmetry measured at the LHC may be statistically limited in a large part of the parameter space. The determination of $F$ in $e p$ collisions is simply obtained by comparing the cross-sections measured in $e^{+} p$ and $e^{-} p$ collisions. The much larger crosssection would make the determination of $F$ possible up to the kinematic limit, for a coupling of $\mathcal{O}(0.1)$. Example asymmetries expected to be measured with $100 \mathrm{fb}^{-1}$ of LHC data, and with $10 \mathrm{fb}^{-1}$ of LHeC data both in $e^{+} p$ and in $e^{-} p$, are shown in Fig. 2. For a coupling $\lambda=0.1$, no information on $F$ can be extracted from the LHC data for a LQ mass above $\sim 700 \mathrm{GeV}$, while the $\mathrm{LHeC}$ can determine $F$ for $\mathrm{LQ}$ masses up to $1 \mathrm{TeV}$.

The LHC discovery of an $e p$ resonance with a mass below about $1 \mathrm{TeV}$, with indications that the coupling $\lambda$ is not too small, would thus be a strong motivation to pursue its study in $e p$ collisions. The LHeC would resolve the remaining ambiguities in the determination of the LQ quantum numbers, and would provide important information on the chiral structure and on the strength of the LQ couplings.

In $R_{p}$-conserved supersymmetry, the dominant process in $e p$ collisions consists of the associated production of a slepton and a squark. The cross-section is above $1 \mathrm{pb}$ as soon as the sum of the slepton and squark masses is below $1 \mathrm{TeV}$. Hence, if squarks are discovered at the LHC with a mass not much higher than the Tevatron reach, selectrons or sneutrinos with a mass up to about $500 \mathrm{GeV}$ may be observed at LHeC, slightly extending beyond the LHC reach.

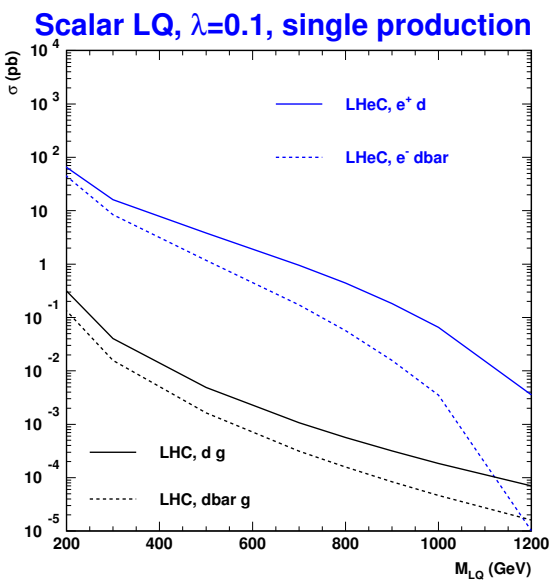

Figure 1: Cross-section for single LQ production at LHC and at LHeC. The example of a scalar LQ coupling to ed is shown here.

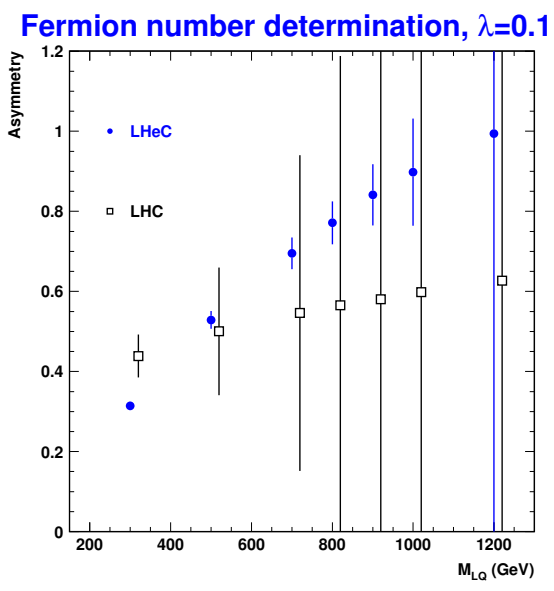

Figure 2: Asymmetries which would determine the fermion number of a LQ, the sign of the asymmetry being the relevant quantity. 


\section{Proton structure and the interpretation of LHC discoveries}

Parton distribution functions (pdfs) are not well know yet in the high $x$ regime, in particular for the down quark and the gluon. Although this limited knowledge would not jeopardize the discovery of a high mass new state at the LHC, as a new $Z^{\prime}$ or $W^{\prime}$ boson, it would limit the information that will come out of the LHC data. For instance, with the ultimate LHC luminosity, a $W^{\prime}$ boson could be discovered with a mass of up to about $6 \mathrm{TeV}$ if its coupling to quarks is similar to that of the standard $W$ boson. For such a high partonic centre-of-mass energy, the uncertainty of the partonic luminosity is of the order of $40 \%$, such that only a rough estimate of the coupling of a $6 \mathrm{TeV} W^{\prime}$ boson to fermions will be possible. An improved knowledge of the high $x$ parton densities, as brought by the LHeC, would be of a high value for the interpretation of the LHC discoveries. As an example, Fig. 3 shows how the uncertainties of the $d$ and gluon densities would be decreased with $10 \mathrm{fb}^{-1}$ of $\mathrm{LHeC}$ data.

In cases where new physics effects would not manifest themselves as a clear "peak" above a background, but as a smooth enhancement or decrease of the measured cross-section as compared to the SM expectation, our limited knowledge of proton pdfs may be more problematic. Such an effect could be observed e.g. in the Drell-Yan mass spectrum at the LHC, and could be due, for example, to the interference of a very heavy $Z^{\prime}$ with the SM bosons, to the exchange of a continuum of Kaluza-Klein states in the presence of large extra-dimensions, etc. Such effects in the di-electron DY spectrum can be generically studied by using an effective Lagrangian density

$$
\mathcal{L}_{C I}=\sum_{i, j=L, R} \epsilon_{i j}^{e q} \frac{4 \pi}{\Lambda^{2}}\left(\bar{e}_{i} \gamma^{\mu} e_{i}\right)\left(\bar{q}_{j} \gamma_{\mu} q_{j}\right)
$$
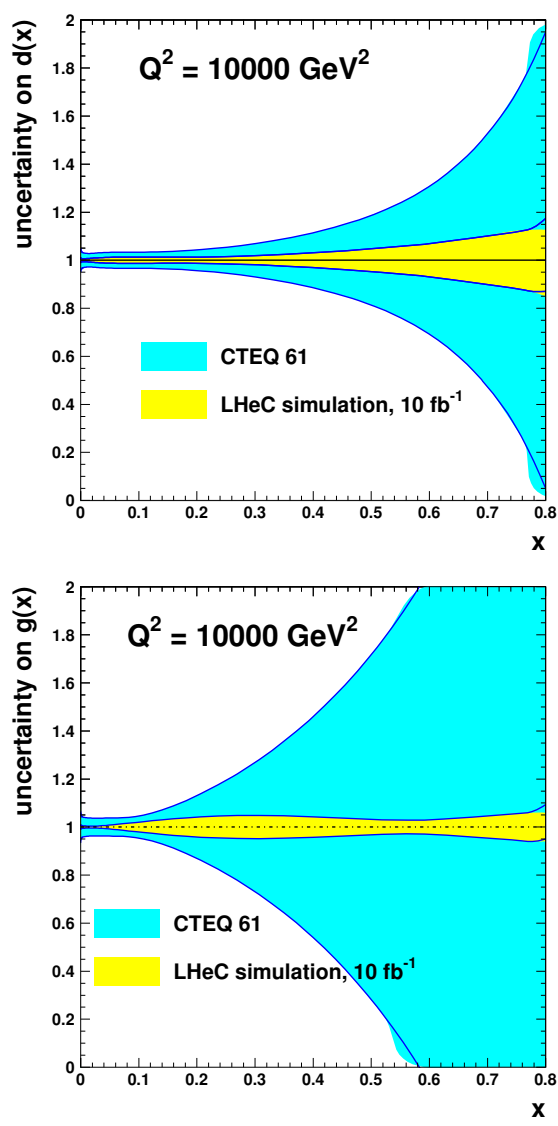

Figure 3: Uncertainty on the down quark and on the gluon densities in the proton, at a scale $Q^{2}=10^{4} \mathrm{GeV}^{2}$. The blue (dark-shaded) error bands show the uncertainties as obtained from the CTEQ 6.1 sets, and the yellow (lightshaded) bands show how these uncertainties would be reduced with $10 \mathrm{fb}^{-1}$ of LHeC data.

in which new physics is embedded in "contact interactions" (CI). Different models can be considered, depending on which terms are present in $\mathcal{L}_{C I}$. The parameters $\epsilon_{i j}= \pm 1$ determine whether the new amplitude(s) interfere constructively or destructively with the SM amplitudes. With $10 \mathrm{fb}^{-1}$ of data, LHeC would probe scales between 25 and $45 \mathrm{TeV}$ depending on the model. The sensitivity of LHC to such eeqq contact interactions is similar. 
Figure 4 shows how the DY crosssection would deviate from the SM value, for three examples of eeqq contact interactions. In the "LL" model considered here, the sum in eq. (1) only involves left-handed fermions and all amplitudes have the same phase $\epsilon$. With only $p p$ data, it will be difficult to determine simultaneously the size of the contact interaction scale $\Lambda$ and the sign of the interference of the new amplitudes with respect to the SM ones: for example, for $\Lambda=20 \mathrm{TeV}$ and $\epsilon=-1$, the decrease of the crosssection with respect to the SM prediction for di-electron masses below $3 \mathrm{TeV}$, which is characteristic of a negative interference, is too small to be firmly established when pdf uncertainties are taken into account. For the same "LL" model, the sign of this interference can be unambiguously determined at LHeC from the asymmetry of $\sigma / \sigma_{S M}$ in $e^{+} p$ and $e^{-} p$ data, as shown in Fig. 5 .

Moreover, with a polarised lepton beam, ep collisions would help determine the chiral structure of the new interaction. More generally, it is very likely that both $p p$ and ep data would be necessary to underpin the structure of new physics which would manifest itself as an eeqq contact interaction. Such a complementarity of $p p$, ep (and also $e e)$ data was studied in [5] in the context of the Tevatron, HERA and LEP colliders.

\section{References}

[1] J.B. Dainton et al., JINST 1 (2006) P10001 [hep-ex/0603016].

[2] P. Newman, these proceedings.

[3] M. Klein, these proceedings.

[4] A. Belyaev et al., JHEP 09005 (2005) [hep-ph/0502067].

[5] A.F. Zarnecki, Eur. Phys. J. C11 539 (1999) [hep-ph/9904334].

[6] Slides:

http://indico . cern. ch/contributionDisplay . py? contribId=260\&sessionId=10\& conf Id=9499

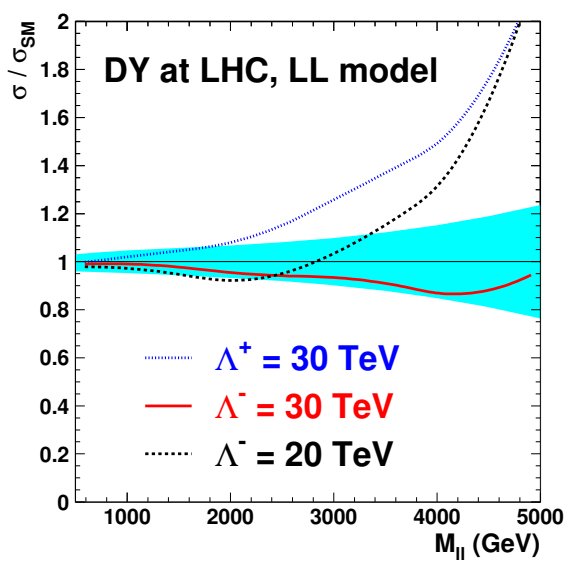

Figure 4: Example deviations, from its SM value, of the Drell-Yan cross-section at LHC as a function of the dilepton mass, in the presence of an eeqq contact interaction.
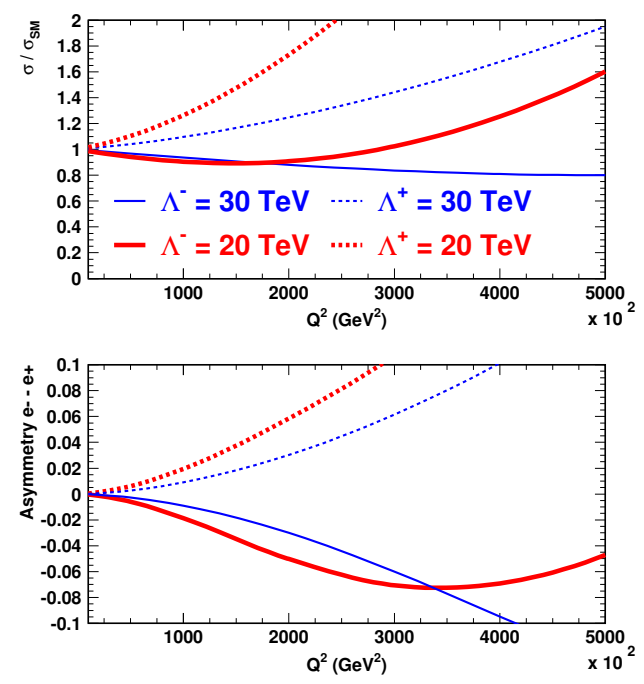

Figure 5: (top) Example deviations of the $e^{-} p$ DIS cross-section at LHeC, in the presence of an eeqq CI. (bottom) Asymmetry of $\sigma / \sigma_{S M}$ between $e^{+} p$ and $e^{-} p$ measurements. 